\title{
The Effects of Central Cholecystokinin Receptor Blockade on Hypothalamic-Pituitary-Adrenal and Symptomatic Responses to Overnight Withdrawal from Alprazolam
}

\author{
James L. Abelson, George C. Curtis, Randolph Nesse, Rosalind Fantone, \\ Robert E. Pyke, and Judith Bammert-Adams
}

Key Words: Drug withdrawal, corticotropin, cortisol, cholecystokinin, panic disorder, CI-988

\section{Introduction}

Abrupt withdrawal from benzodiazepines (BZs) produces anxiety and potently activates the hypothalamic-pituitary-adrenal (HPA) axis in animals (Eisenberg 1987; Owens et al 1991). Alcohol withdrawal similarly activates the HPA axis in humans (Adinoff et al 1991; von Bardeleben et al 1989). The human HPA response to BZ withdrawal has been little studied (Adam et al 1984), but the animal and human alcohol data predict activation.

Abrupt BZ withdrawal also produces increases in hippocampal and cortical cholecystokinin (CCK) receptor number (Harro et al 1990) and in pre-proCCK mRNA (Rattray et al 1993). CCK agonists can induce anxiety and activate hippocampal neurons; both of these effects are antagonized by BZs (Bradwejn and de Montigny 1984; de Montigny 1989). CCK also activates the HPA axis (Abelson et al 1991), whereas BZs inhibit it (Owens et al 1991). CCK and BZs thus appear to interact antagonistically in modulating anxiety and the stress axis, raising the possibility that BZ withdrawal-induced anxiety and HPA axis activation could be me-

From the University of Michigan Department of Psychiatry, Anxiety Disorders Program (JLA, GCC, RN, RF) and the Parke-Davis Pharmaceutical Research Division, Warner-Lambert Company (REP, JB-A), Ann Arbor, Michigan.

Address reprint requests to James L. Abelson, M.D., Ph.D., Rm C435, Med Inn Bldg/0840, 1500 E. Medical Center Drive, Ann Arbor, MI 48109-0840; Fax: 313-936-7868.

Received January 28, 1994; revised July 8, 1994. diated by CCK. If so, then a CCK receptor antagonist may be able to block the anxiety and HPA axis activation produced by BZ withdrawal. A selective CCK-B receptor antagonist (CI-988) has in fact been shown to block BZ withdrawal anxiety in animal models (Costall et al 1991; Singh et al 1992).

We explored the HPA responses to BZ withdrawal in humans and the effects of a CCK antagonist (CI-988) on this response and on withdrawal symptoms by using overnight withdrawal from alprazolam. Chronic alprazolam users frequently experience withdrawal symptoms upon missing a single dose, so we could study the early stages of withdrawal by postponing their usual morning dose. Because of safety concerns in testing a new class of medication in a new paradigm, we chose a low dose of CI-988 for this preliminary study and were particularly interested in neuroendocrine effects.

\section{Methods}

Six patients under long-term treatment with alprazolam for panic disorder were studied after informed consent was obtained. Each subject was studied three times, separated by 1 week, under the following conditions 1) nonwithdrawal (usual morning dose of alprazolam given), 2) untreated withdrawal (AM alprazolam postponed, placebos given), and 3) treated withdrawal (AM alprazolam postponed, $25 \mathrm{mg}$ of CI-988 given). Because full randomization of order with three conditions requires a larger sample than was feasible for this pilot study and because we wished to directly compare CI-988 to placebo, these latter two conditions were presented in a double-blind, cross-over design with constrained 
random assignment to order so each order was equally represented. The nonwithdrawal condition was always given first and primarily provided control data for neuroendocrine analyses. Presenting the nonwithdrawal condition first prevented subject attrition by avoiding a stressful withdrawal experience upon first exposure to the laboratory. It also ensured that predictable order effects (increased anxiety and HPA activation during first exposure to the laboratory; Abelson et al 1994), would not confound hypothesis confirmation, in that alprazolam would have to overcome these novelty effects to confirm our prediction that nonwithdrawal would be less activating than withdrawal.

All subjects were medically healthy, nondepressed (Ham-D $<18$ ), free of other psychotropic medication or drugs of abuse (confirmed by urine drug screen), and of non-child-bearing potential. Their mean age was 43.2 yr (range: $30-60$ ). Diagnoses were confirmed by a structured clinical interview (SCID). All had a primary diagnosis of panic disorder, but five had additional SCID diagnoses. Two subjects had simple phobias (heights, elevators). One of these also had generalized anxiety disorder (GAD). Two subjects had histories of substance abuse (alcohol, substance free for 2 years; marijuana, substance free for 10 years). One of these (the former alcohol abuser) also had obsessive-compulsive disorder. One subject had social phobia, GAD, and a history of a major depressive episode. Entry criteria required at least 6 months on at least 3 times/day dosing of a $\mathrm{BZ}$ equivalent to at least 3 $\mathrm{mg} /$ day of alprazolam, and subjective report of withdrawal symptoms within 12 hours after a dose (anxiety symptoms upon missing a dose or on arising after a night of sleep). Subjects had been on BZs for a mean of $5.6 \mathrm{yr}$ (range: $8 \mathrm{mo-10} \mathrm{yr}$ ); all but one had taken alprazolam daily for more than 4 years. The only exception had been on alprazolam daily for 2 months after 6 months on daily clorazepate. Mean dose at time of study was $4 \mathrm{mg} /$ day (range: 3-6).

All patients underwent a trial withdrawal at home 1 week prior to study. They held their morning dose until they had gone without drug for 8-16 hours, with hourly telephone monitoring of withdrawal symptoms by the study psychiatrist (JLA). The approximate time of onset of withdrawal symptoms was determined for each patient, and alprazolam was resumed before symptoms became severe. Patients reported to a Clinical Research Center (CRC) on the evening prior to study, where they received a last dose of alprazolam at a time determined by their preadmission trial to be likely to produce mild symptoms of withdrawal by $8 \mathrm{AM}$ the next day. They remained in the CRC overnight, with minimal activity and fasting from 11 PM until noon the next day. Intravenous access was established by 7:00 AM. Blood samples were obtained at 7:30, 7:45, 8:00 AM, and every $30 \mathrm{~min}$ thereafter until noon. Symptoms and vital signs were monitored hourly. Heart rate (HR), systolic blood pressure (SBP), and diastolic blood pressure (DBP) were measured using an Air-Shields automated monitor. Anxiety was measured using a visual analogue scale (VAS), and withdrawal symptoms were monitored with a revised form of the Liebowitz Withdrawal Symptom Checklist (WDS) (Noyes et al 1991).

Alprazolam or alprazolam placebo were administered singleblindly at 7:00 AM. CI-988 or CI-988 placebo were administered at 8:00 AM. On the first visit to the CRC, all subjects received their regular alprazolam dose at 7:00 $\mathrm{AM}$ and a placebo at 8:00 AM (nonwithdrawal condition). On their second and third visits they received placebo at 7:00 AM and were in early withdrawal when they received double-blind administration of CI-988 or placebo at 8:00 AM. All patients resumed their usual alprazolam dosing at noon.

Adrenocorticotropic hormone (ACTH) was measured by radioimmunoassay, with a sensitivity of $6 \mathrm{pg} / \mathrm{ml}$ and intraassay and interassay coefficients of variation (CVs) of less than $10 \%$. Cortisol was measured by high-performance liquid chromatography, with a sensitivity of $0.1 \mu \mathrm{g} / \mathrm{dl}$ and CVs of $<6 \%$.

Areas under the curve (AUC, by trapezoidal approximation) for ACTH and cortisol levels from 9:00 AM until noon were used as the measures of HPA axis activity for hypothesis testing. These provide the best estimate of overall axis activity during a time period that allows for the onset of action of CI-988 and alprazolam and captures the fullest degree of withdrawal to which we subjected the subjects. Symptom measures for hypothesis testing were the peak VAS anxiety rating and the peak total WDS score between 9:00 AM and noon. Peak scores were used to capture maximum symptom development from symptom response curves with considerable intersubject variability in time course. The following four predictions were tested: 1) Symptoms will be greater during untreated withdrawal than on the nonwithdrawal day (placebo vs. alprazolam); 2) ACTH and cortisol secretion will be greater during untreated withdrawal than on the nonwithdrawal day; 3) symptoms will be less during treated withdrawal than during untreated withdrawal (CI-988 vs. placebo); and 4) ACTH and cortisol secretion will be less during treated withdrawal than during untreated withdrawal. All hypotheses make directional prediction, and hypothesis testing comparisons were planned prior to data collection, so we used one-tailed, paired $t$ tests with .05 level of significance.

\section{Results}

Means, standard deviations, and $t$ test results are presented in Table 1. Hypotheses 1, 2, and 4 were supported. Alprazolam withdrawal (holding the usual morning dose in chronically treated patients) produced significant anxiety and withdrawal symptoms and activated the HPA axis. CI-988 successfully attenuated the cortisol response to alprazolam withdrawal. The ACTH response was also reduced, though not significantly. Hypothesis 3 was not supported. Following CI-988 administration, withdrawal symptoms were not reduced but were nonsignificantly elevated.

To follow up the significant effects of withdrawal and CI-988 treatment on cortisol secretion, a full set of paired $t$ tests was conducted, comparing all pairings of nonwithdrawal, untreated withdrawal, and treated withdrawal at every time point. The complete cortisol response curve and results of these additional comparisons are presented in Figure 1. There were no differences between conditions at baseline. The alprazolam (nonwithdrawal) and CI-988 conditions did not differ significantly from each other at any time point. Both treated conditions differed from untreated withdrawal at the majority of time points between 9:00 AM and noon, when withdrawal effects were expected to be most evident.

To verify that there were no baseline differences between conditions, we conducted single-factor analyses of variance (ANOVAs) on baseline ACTH and cortisol levels (mean of the 7:30, 7:45, and 8:00 AM measures) and baseline symptom ratings (7:30 AM 
Table 1. Mean ( \pm SD) Symptom and Hormone Levels in Three Treatment Conditions, With Statistical Comparisons (Paired $t$ Tests)

\begin{tabular}{lccccc}
\hline & $\begin{array}{c}\text { Nonwithdrawal } \\
\text { (Alprazolam) }\end{array}$ & $\begin{array}{c}\text { Untreated } \\
\text { withdrawal } \\
\text { (Placebo) }\end{array}$ & $\begin{array}{c}\text { Treated } \\
\text { withdrawal } \\
\text { (Cl-988) }\end{array}$ & $\begin{array}{c}\text { Alprazolam } \\
\text { vs. } \\
\text { placebo }\end{array}$ & $\begin{array}{c}\text { CI-988 } \\
\text { vs. } \\
\text { placebo }\end{array}$ \\
\hline VAS anxiety (peak) & $35.0 \pm 16.3$ & $55.2 \pm 30.8$ & $65.8 \pm 32.3$ & $t=2.6, p=.025$ & $t=0.6, p=.55$ \\
WDS total (peak) & $3.2 \pm 2.6$ & $9.7 \pm 7.6$ & $13.2 \pm 9.5$ & $t=2.9, p=.018$ & $t=1.0, p=.37$ \\
ACTH (AUC) & $3597.5 \pm 1350.6$ & $5100 \pm 2510.6$ & $4500 \pm 2470.8$ & $t=2.5, p=.026$ & $t=0.9, p=.21$ \\
Cortisol (AUC) & $1775 \pm 373.2$ & $2393 \pm 501.8$ & $1971 \pm 396.4$ & $t=4.4, p=.003$ & $t=6.6, p=.0006$ \\
\hline
\end{tabular}

VAS = visual analogue scale; WDS = Liebowitz Withdrawal Symptom Checklist; ACTH = adrenocorticotropic hormone; AUC $=$ area under the curve

VAS/anxiety and WDS total). There were no significant differences among the three treatment conditions in these analyses.

To determine whether patients found the experimental situation less stressful as they gained more experience with it, we compared HPA and symptom responses on visits 2 and 3 , since during both of these visits patients were put into alprazolam withdrawal. Patients experienced significantly fewer symptoms of anxiety and withdrawal the second time they were withdrawn $(t=3.5, p=.02$ for VAS anxiety; $t=2.5, p=.05$ for WDS total score). ACTH and cortisol did not differ between visits ( $t<0.6, p>.50$ for both).

Multiple analyses could detect no significant effect of CI-988 on HR or BP. Data from CI- 988 and placebo days were combined and compared to alprazolam day data to search for cardiovascular effects of withdrawal. The only detectable trend was for elevated SBP during "late" withdrawal (11:00 AM to noon) compared to the same time period on nonwithdrawal day $(t=2.1, p=0.09)$.

\section{Discussion}

These data must be considered preliminary because of the small sample and use of only a single, low dose of CI-988. Generalization to a nonpatient population may also not be appropriate, since

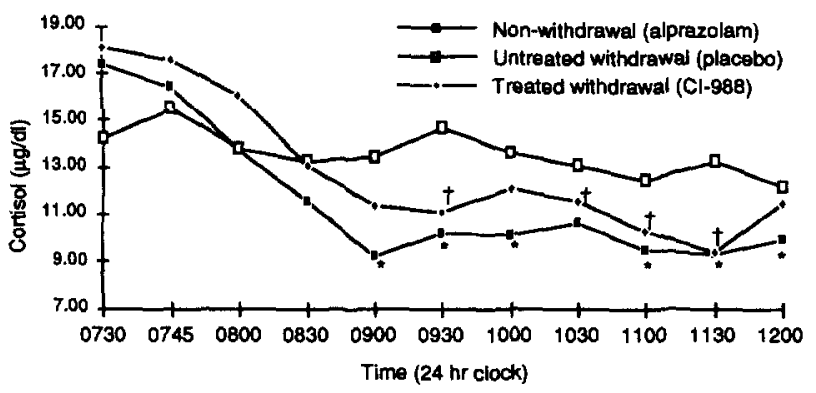

Figure 1. Cortisol responses in chronic alprazolam users to untreated overnight withdrawal (placebo), withdrawal treated with CI988, and nonwithdrawal (alprazolam). Alprazolam/placebo was given at 0700; C1988/placebo was given at 0800 . Untreated withdrawal produced significantly $(p<.05)$ elevated cortisol levels relative to alprazolam at points marked with asterisks $(*)$. Untreated withdrawal produced significantly elevated cortisol levels relative to CI988 at points marked with daggers ( $\dagger$ ). Alprazolam and CI988 treatments did not differ significantly from each other at any time points. patients with panic disorder may have altered sensitivities to withdrawal anxiety, HPA axis activation, and CCK or BZ receptor stimulation. With these caveats in mind, however, the data do support the hypothesis that BZ withdrawal activates the HPA axis. Normal diurnal variation should produce falling ACTH and cortisol levels during the morning hours. When chronic users of alprazolam were denied their usual morning dose of this short-acting $\mathrm{BZ}$, their ACTH and cortisol levels did not follow this normal pattern but instead remained level throughout the morning. When their morning alprazolam was administered, levels did fall and were significantly lower than in the "withdrawn" state. The magnitude of the withdrawal effect was likely greatly underestimated in this study, since HPA axis activity was probably elevated on the nonwithdrawal day by a first-visit "novelty" effect (Abelson et al 1994) and since, for ethical reasons, we were unwilling to hold alprazolam for more than 16 hours. The appearance of cardiovascular effects (SBP) late in our withdrawal period (11:00 AM to noon) suggests that, though our subjects were already experiencing withdrawal symptoms at 8:00 AM, they may have been just beginning to enter a more active state of physiological withdrawal when we terminated our observation period at noon.

An alternative interpretation of the data is available, in that lower cortisol levels in the nonwithdrawal condition could reflect alprazolam's ability to acutely depress HPA activity rather than the ability of withdrawal to elevate it. Though acute administration of BZs has been shown to decrease ACTH and cortisol levels, the literature is not entirely consistent (Schuckit et al 1992), and the effects of acute administration after long-term, daily administration have not been studied. Separate data from our laboratory (unpublished) indicate that panic patients on alprazolam do not differ from untreated normal controls in their decline in cortisol over the morning hours and support our conclusion that the differences between withdrawal and nonwithdrawal in the current data set are due to the failure of withdrawing patients to show the normal diurnal decline.

Administration of the CCK-B antagonist CI-988 successfully attenuated withdrawal-induced HPA axis activation in this study, even though it failed to reduce withdrawal anxiety. This finding strongly supports our hypothesis, based on work with a CCK-B agonist (Abelson et al 1991), that CCK plays a physiological role in regulating the HPA axis and does so independently of its effects on anxiety and subjective distress. CI-988 was able to reduce cortisol levels, compared to placebo, even though withdrawal symptoms and anxiety levels were nonsignificantly higher in the CI-988 condition. Its success in inhibiting the HPA axis is particu- 
larly impressive in light of the relatively low dose chosen for this pilot study and its pharmacokinetics ( $T_{\max }$ of 2-3 hours), which should have produced maximal effects during only a part of the period studied. That the HPA effect is more readily seen for cortisol than for ACTH may be because cortisol provides a more reliable indicator of stress axis activity when samples are obtained only every $\mathbf{3 0}$ minutes. More frequent sampling is needed to fully capture ACTH secretory episodes, since these are pulsatile and the ACTH half-life is quite short. It is also possible that CI-988 has some direct effect at the adrenal level. Our data are consistent with the BZ literature, which has more readily demonstrated effects of BZs on cortisol levels than ACTH levels (Schuckit et al 1992).

Though CI-988 appeared to suppress cortisol to nearly the same extent as alprazolam, these data must be interpreted with great caution. The study was not designed to directly compare the two drugs, but rather to test specific hypotheses by comparing both to placebo. Order effects are not controlled in an alprazolam-CI-988 comparison, in that alprazolam was always given first. Novelty effects may have made CI-988 look better relative to alprazolam by exaggerating symptom levels and HPA activity in the alprazolam condition. Differing pharmacokinetics, however, worked to the advantage of alprazolam in that its earlier administration and quicker onset of action should have led to greater pharmacological activity over the period studied. Further work with other designs is needed.

Our anxiety and withdrawal symptom data must also be interpreted cautiously. Because of the low dose of CI-988 used and the

\section{References}

Abelson JL, Nesse RM. Vinik A (1991): Stimulation of corticotropin release by pentagastrin in normal subjects and patients with panic disorder. Biol Psychiatry 29:1220-1123.

Abelson Л, Nesse RM, Vanik A (in press): Pentagastrin infusions in patients with panic disorder. II Neuroendocrinology. Biol Psychiatry.

Adam K, Oswald I, Shapiro C (1984): Effects of loprazolam and of triazolam on sleep and overnight urinary cortisol. Psychopharmacology 82:389-394.

Adinoff B, Risher-Flowers D, De Jong J, et al (1991): Disturbances of hypothalamic-pituitary-adrenal axis functioning during ethanol withdrawal in six men. Am $J$ Psychiatry 148:1023-1025.

Bradwejn J, de Montigny C (1984): Benzodiazepines antagonize cholecystokinin-induced activation of rat hippocampal neurons. Nature 312:363-364.

Costall B, Domeney AM, Hughes J, Kelly ME, Naylor RJ, Woodruff GN (1991): Anxiolytic effects of CCK-B antagonists. Neuropeptides 19(suppl):65-73.

de Montigny C (1989): Cholecystokinin tetrapeptide induces panic-like attacks in healthy volunteers. Arch Gen Psychiatry 46:511-517.

Eisenberg RM (1987): Diazepam withdrawal as demonstrated by changes in plasma corticosterone: A role for the hippocampus. Life Sci 40:817-825.

Harro J, Lang A, Vasar E (1990): Long-term diazepam treatment produces changes in cholecystokinin receptor binding in rat brain. Eur J Pharmacol 180:77-83. combination of a small sample and the strong confounding effect of order on symptom responses to withdrawal, the data can shed no real light on the effect of CI-988 on withdrawal anxiety. Follow-up studies using larger samples and parallel group designs are planned. Our demonstration of CI-988's ability to reduce "stress"induced cortisol release is extremely encouraging, since in early animal studies of other agents this ability consistently predicted anxiolytic efficacy (Lahti and Barsuhn 1974).

Finally, we want to call attention to the striking effect of having "been through it once" in reducing the anxiety and symptom response to the withdrawal experience. Both subjective observation and the data analyses readily demonstrated that the second experience with withdrawal was far less distressing than the first. Whether this effect reflects panic patients' hypersensitivity to "novelty" (in this case the experience of withdrawal symptoms in a novel research environment) or the rapidity and power of desensitization in these patients, we believe that it may reflect a critical underlying feature of panic disorder. It deserves more extensive exploration in its own right and must be considered in all studies of panic that expose patients to novel experiences and environments.

This research was supported in part by Clinical Research Center Grant \#M01RR00042 and by a grant from Parke-Davis.
Lahti RA, Barsuhn C (1974): The effect of minor tranquilizers on stress-induced increases in rat plasma corticosteroids. Psychopharmacologia 85:215-220.

Noyes R, Garvey MJ, Cook B, Suelzer M (1991): Controlled discontinuation of benzodiazepine treatment for patients with panic disorder. Am J Psychiatry 148:517-523.

Owens MJ, Vargas MA, Knight DL, Nemeroff CB (1991): The effects of alprazolam on corticotropin-releasing factor neurons in the rat brain: Acute time course, chronic treatment, and abrupt withdrawal. J Pharmacol Exp Ther 258:349-356.

Rattray M, Singhvi S, Pei-Ying W, Andrews N, File SE (1993): Benzodiazepines increase preprocholecystokinin messenger RNA levels in rat brain. Eur J Pharmacol 245:193-196.

Schuckit MA, Hauger R, Klein JL (1992): Adrenocorticotropin hormone response to diazepam in healthy young men. Biol Psychiatry 31:661-669.

Singh L, Field MJ, Vass CA, Hughes J, Woodruff GN (1992): The antagonism of benzodiazepine withdrawal effects by the selective cholecystokinin B receptor antagonist CI-988. Br J Pharmacol 105:8-10.

von Bardeleben U, Heuser I, Holsboer F (1989): Human CRH stimulation response during acute withdrawal and after medium-term abstention from alcohol abuse. Psychoneuroendocrinology 14:441-449. 